\title{
USING CHIMERIC USERS TO CONSTRUCT FUSION CLASSIFIERS IN BIOMETRIC AUTHENTICATION TASKS: AN INVESTIGATION
}

\author{
Norman Poh and Samy Bengio \\ IDIAP Research Institute, CP 592, 1920 Martigny, Switzerland, and \\ Ecole Polytechnique Fédérale de Lausanne (EPFL), Lausanne, Switzerland. \\ \{norman, bengio\}@idiap.ch
}

\begin{abstract}
Chimeric users have recently been proposed in the field of biometric person authentication as a way to overcome the problem of lack of real multimodal biometric databases as well as an important privacy issue - the fact that too many biometric modalities of a same person stored in a single location can present a higher risk of identity theft. While the privacy problem is indeed solved using chimeric users, it is still an open question of how such chimeric database can be efficiently used. For instance, the following two questions arise: i) Is the performance measured on a chimeric database a good predictor of that measured on a real-user database?, and, ii) can a chimeric database be exploited to improve the generalization performance of a fusion operator on a real-user database?. Based on a considerable amount of empirical biometric person authentication experiments $(21$ real-user data sets and up to $21 \times 1000$ chimeric data sets and two fusion operators), our previous study [1] answers no to the first question. The current study aims to answer the second question. Having tested on four classifiers and as many as 3380 face and speech bimodal fusion tasks (over 4 different protocols) on the BANCA database and four different fusion operators, this study shows that generating multiple chimeric databases does not degrade nor improve the performance of a fusion operator when tested on a real-user database with respect to using only a real-user database. Considering the possibly expensive cost involved in collecting the real-user multimodal data, our proposed approach is thus useful to construct a trainable fusion classifier while at the same time being able to overcome the problem of small size training data.
\end{abstract}

\section{INTRODUCTION}

Biometric authentication is a problem of verifying an identity claim using a person's behavioral and physiological characteristics. While this can be achieved based on a single modality (voice or face prints for instance), the current literature provides several approaches towards studying fusion of such modalities for better performance and robustness. One practice is to construct a large database containing several biometric traits for each user. This, however, can be very time-consuming, expensive, and of ethical concern. Another practice is to combine biometric modalities of a database with biometric

The authors thank the following people who helped brainstorming the subject: Johnny Mariéthoz, and many participants of MLMI'05 and IDIAP's TAM. This work was supported in part by the IST Program of the European Community, under the PASCAL Network of Excellence, IST-2002-506778, funded in part by the Swiss Federal Office for Education and Science (OFES) and the Swiss NSF through the NCCR on IM2. This publication only reflects the authors' view. modalities of another biometric database. Since both databases do not necessarily contain the same users, such combination results in chimeric users. From the experimental point of view, these biometric modalities belong to the same person. While this practice is commonly used in the multimodal literature, e.g., [2,3] among others, it was questioned whether this was a right thing to do or not during the 2003 Workshop on Multimodal User Authentication [4].

There are at least two arguments that justify the use of chimeric users, i.e., i) modality independence assumption - that two or more biometric traits of a single person are often assumed independent of each other; and ii) privacy issue - participants in the multimodal biometric experiments are often not ready to let institutes keep record of too much of their personal information (raw biometric data) at the same place. If such information is misused, it could be dangerous, e.g., identity theft. It is for this same reason that processed biometric features are preferred for storage to raw biometric data. Note that the first argument is technical while the second one is ethical. Although both arguments are equally important, the second one is beyond an experimenter's control and is related to the usage policy of the database. For instance the policy should address who can use the database and how it should be used. When a database is carefully designed to protect the participants' privacy right, this issue should be resolved. For this reason, this paper focuses on the first argument.

In our previous study [1], we addressed the question: "Is the performance measured on a chimeric database a good estimator of that measured on a real-user database?". Having conducted a considerable amount of empirical experiments (21 real-user data sets and up to $21 \times 1000$ chimeric data sets on two fusion operators, the answer is no. In other words, the performance based on a chimeric database can possibly be biased. This means that, for instance, one cannot claim that novel algorithm $\mathrm{A}$ is better than state-of-the-art algorithm $\mathrm{B}$ on a real multimodal biometric authentication task if the comparison was conducted on a chimeric database. A similar investigation was reported in [5] with the conclusion that favors the use of chimeric users. It should be pointed out that these studies were undertaken with the following differences: (i) the former was tested on 21 fusion tasks whereas the latter was tested on two fusion tasks (clean and noisy); (ii) the former engaged in a standard hypothesis test whereas the latter did not - only the mean DET curves derived from both real and chimeric databases were visually compared; (iii) the former is based on a threshold dependent assessment - whereby a threshold is optimized a priori on a development (training) set and a performance is measured on an evaluation (test) set using the chosen threshold; a similar assessment as the yearly NIST evaluation protocols [6]) - whereas the latter is based on a threshold free assessment via a DET curve; (iv) two fusion operators are considered 

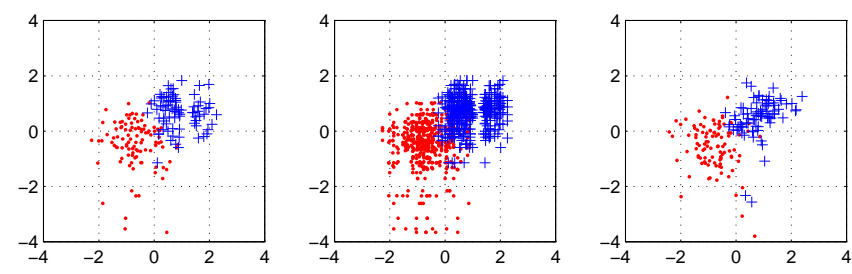

Fig. 1. Left: an original bimodal fusion training data set whose $\mathrm{x}$-axis is a speech expert score and $\mathrm{y}$-axis is a face expert score. Center: a bimodal fusion training data set generated using chimeric users. Right: a bimodal real-user test data set. This is a typical example among the 3380 fusion tasks taken from the BANCA database. In each figure, crosses (upper right cluster) denote client accesses and dots (lower left cluster) denote impostor accesses.

in the former and only one considered in the latter; and (v) a bootstrap procedure was used in the latter to estimate the distribution of performance on the real-user database and the former did not ${ }^{1}$. While most differences are methodological, it should be remarked that our findings show that only approximately a third of 21 fusion data sets, independent of the fusion classifier used, reports inconsistency of performance between the real-user and chimeric databases. Hence, the inconsistency may very well not be visible with only 2 experiments.

This paper addresses another issue with respect to chimeric users: "Can a chimeric database be exploited to improve the generalization performance of a fusion operator on a real-user database?". Very often, due to lack of training data, a fusion operator has very limited amount of data for training. Hence, by using chimeric database, one can generate much more data to train the fusion classifier that would then be assessed on real multimodal user scores. If this is the case, then, even if the performance measured on a chimeric database is biased as in [1], a chimeric database is still at least useful for other purposes such as to help construct a fusion classifier. To verify this hypothesis, we limit our scope to studying such effect to bimodal as generalization to more than two modalities is direct.

This paper is organized as follows: Section 2 contains a description on the general methodology used; Section 3 describes the BANCA database used; Section 4 presents the four fusion classifiers used; and Section 5 presents the experimental outcomes.

\section{METHODOLOGY}

To illustrate the idea, we first plot a bimodal fusion training and test sets in the left and right panels of Figure 1, respectively. By random mix-and-match of (scores of) modalities according to different identities, we obtained a much larger training set as shown in the middle panel of Figure 1.

Although this methodology is rather simple, there is still a fundamental question of how many chimeric users are necessary. Suppose that there are $N$ real users for which we recorded 2 modalities. Then, in theory, in order to construct a bimodal chimeric database, one can generate up to $N \times(N-1)$ chimeric users (by excluding the $N$ real users). Our initial experiments with $N, 2 N, 3 N, \ldots,(N-$ 1) $\times N$ (as a multiple of the user size) show that the number of users

\footnotetext{
${ }^{1}$ Recognizing that this issue is important, our on-going work takes into account of such information but the experimental outcome does not change the conclusion reported in [1].
}

has not much effect on the performance. We thus fixed this multiple factor to 10 so that the fusion constructed on chimeric users had 10 times more data than that trained on real users.

\section{DATABASE}

We used the real bimodal face and speech BANCA database. Some of the scores were obtained from [7] ${ }^{2}$ while the rest of the data, based on face systems, are taken from [8]. These systems contain experimental as well as the state-of-the-art systems based on Principal Component Analysis, Linear Discriminant Analysis, Gaussian Mixture Models, Hidden Markov Models, Support Vector Machines, Normalized Correlation, etc, to name a few. In the BANCA database, there are 7 different protocols, of which we chose four: $\mathrm{Mc}, \mathrm{Ua}, \mathrm{Ud}$ and P. The first three represent matched controlled, unmatched adversed, unmatched degraded scenarios, respectively. The last one is a pooled scenario containing the first three. A matched scenario implies that the mismatch between a training and a test set is minimal (due to using the same type of microphone, video camera and data acquired in similar and clean conditions). There are two unmatched scenarios: adversed and degraded. The former refers to the mismatch due to different acquisition environment whereas the latter refers to using a degraded acquisition device (by simulation). There are five language subsets but only the English subset is used in this study. By combining a speech-based biometric system with a facebased biometric system, the first three protocols contain 840 fusion tasks whereas the last one contains 860 . In the BANCA protocols, two groups of users are distinguished and are labeled by g1 and g2. We used $\mathrm{g} 1$ as a development (training) set and $\mathrm{g} 2$ as an evaluation (test) set; hence, while g1 was modified to create chimeric users, g2 was kept with real users only, in order to be able to assess performance on real users.

\section{FUSION CLASSIFIERS AND THRESHOLD ESTIMATION}

Four classifiers are used, namely Logistic Regression (LR) [9], Gaussian Mixture Model (GMM) with dependent assumption [10], GMM with independent assumption and the mean operator. Note that the LR classifier used here is more general than the one used in [9] (which assumes common covariance of both client and impostor distributions) but rather the standard approach as described in [11]. Let $\boldsymbol{y} \equiv\left[y_{1}, \ldots, y_{M}\right]^{T}$ be a vector of scores consisting of $M$ biometric modalities. The LR classifier has the following form:

$$
y_{L R} \equiv P(C \mid \boldsymbol{y})=\frac{1}{1+\exp (-g(\boldsymbol{y}))},
$$

where

$$
g(\boldsymbol{y})=\sum_{i=1}^{M} \beta_{i} y_{i}+\beta_{0} .
$$

We used an implementation described in [12]. The classical approach of using GMM in classification [10, Chap. 2] is to establish a Log-likelihood ratio (LLR) test between the client and impostor classes, i.e., $k=\{C, I\}$. The LLR takes the following forms:

$$
y_{d e p} \equiv \log \frac{p(\boldsymbol{y} \mid C)}{p(\boldsymbol{y} \mid I)}
$$

\footnotetext{
${ }^{2}$ Available at "ftp://ftp.idiap.ch/pub/bengio/banca/banca_scores"
} 
for the dependent assumption and

$$
y_{\text {indep }}=\log \frac{\prod_{i} p\left(y_{i} \mid C\right)}{\prod_{i} p\left(y_{i} \mid I\right)}
$$

for the independent assumption. The approximations to Eqn. (1) and Eqn. (2) using GMM can be written as follow:

$$
\begin{aligned}
\hat{p}(\boldsymbol{y} \mid k) & =\sum_{c}^{N_{c}} w_{c}^{k} \mathcal{N}\left(\boldsymbol{y} \mid \boldsymbol{\mu}_{c}^{k}, \boldsymbol{\Sigma}_{c}^{k}\right), \\
\hat{p}(y \mid k) & =\sum_{c}^{N_{c}} w_{c}^{k} \mathcal{N}\left(y \mid \mu_{c}^{k},\left(\sigma_{c}^{k}\right)^{2}\right),
\end{aligned}
$$

for any $y \in\left\{y_{i} \mid i=1, \ldots, M\right\}$, respectively, where, the $c$-th component of the class conditional (denoted by $k$ ) mean vector is $\boldsymbol{\mu}^{k}=$ $\left[\mu_{1}^{k}, \ldots, \mu_{M}^{k}\right]^{T}$ and its covariance matrix of dimension $M \times M$ is $\Sigma_{c}^{k}$. The mean and variance of $p(y \mid k)$ are defined similarly except that it is single dimensional. The GMM parameters can be optimized using the Expectation-Maximization algorithm [10] for instance and the number of components can be tuned by validation or optimization of a criterion, e.g., minimum description length [13]. Finally, the fused score using the mean operator has the following form:

$$
y_{\text {mean }}=\frac{1}{M} \sum_{i=1}^{M} \frac{y_{i}-B_{i}}{A_{i}},
$$

where $B_{i}$ and $A_{i}$ are called a bias and a scaling factor, respectively. In our implementation, both parameters are estimates of mean and standard deviation from the training scores, respectively. The resultant normalized $y_{i}$ score is sometimes called a z-score.

Note that the above fusion classifiers do not include a thresh$\mathrm{old}^{3}$. The complete model has the following decision function:

$$
\operatorname{decision}(y)= \begin{cases}\text { accept } & \text { if } y>\Delta \\ \text { reject } & \text { otherwise, }\end{cases}
$$

where $\Delta$ is a global decision threshold and $y$ in our context is any of the combined scores $y \in\left\{y_{L R}, y_{\text {dep }}, y_{\text {indep }}, y_{\text {mean }}\right\}$ discussed before. In a threshold-dependent assessment based on Expected Performance Curve [14], the $\Delta$ is chosen to minimize, on a separate development set, the following criterion, known as Weighted Error Rate (WER),

$$
\Delta_{*}=\arg \min _{\Delta} \operatorname{WER}_{\alpha}(\Delta)
$$

where

$$
\operatorname{WER}_{\alpha}(\Delta) \equiv \alpha \operatorname{FAR}(\Delta)+(1-\alpha) \operatorname{FRR}(\Delta),
$$

and $\alpha$ ranges from 0 to 1 . This parameter balances between the costs between FAR and FRR estimated from a development set. Note that although not having the exact same formulation, similar criteria were employed in the yearly NIST speaker evaluation plans [6] and the BANCA protocols [15]. Using this threshold, we can then evaluate WER for several values of $\alpha$ on the evaluation set. This enables us to obtain unbiased estimates of performance since all hyper-parameters of the fusion operator, including the threshold, are selected on the development or a separate validation set. Note that only a priori performances are reported here.

\footnotetext{
${ }^{3}$ The LR classifier has a bias but it is not used since the algorithm does not explicitly optimize Equal Error Rate or any authentication-related performance.
}

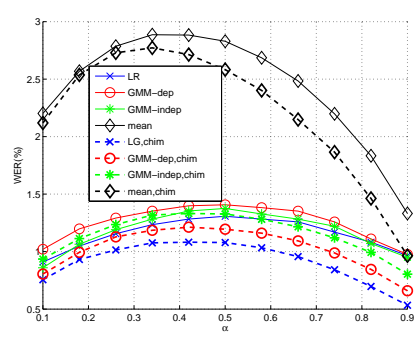

(a) $\mathrm{Mc}$

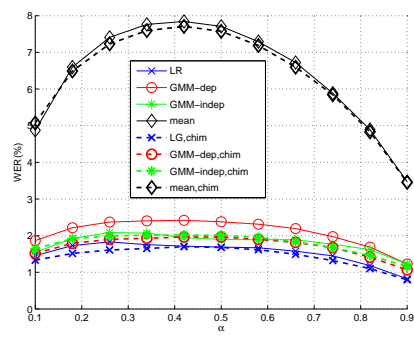

(c) $\mathrm{Ud}$

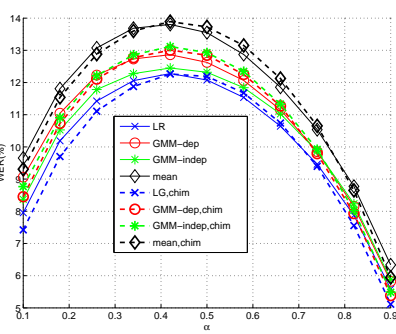

(b) $\mathrm{Ua}$

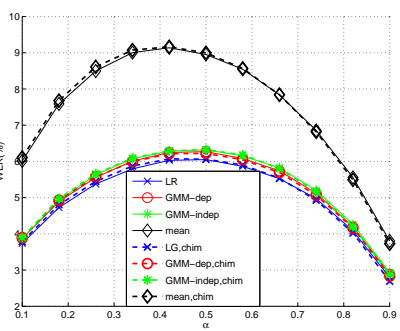

(d) $\mathrm{P}$
Fig. 2. WER versus $\alpha$ (the lower the better) on (a) Mc (840 fusion sets $\times 4$ fusion operators), (b) Ua $(840 \times 4)$, (c) Ud $(840 \times 4)$ and (d) $\mathrm{P}(860 \times 4)$ protocols. The four fusion operators are: logistic regression (cross), GMM with dependent assumption (circle), GMM with independent assumption (asterisk) and the mean operator (diamond). Comparison should be made between a thin continuous line and a thick dashed line.

\section{EXPERIMENTAL RESULTS}

Figure 2 shows pooled EPC curves of four fusion classifiers trained on a real-user development set and an augmented chimeric-user development set having 10 times more data than the former development set. This gives $4 \times 2=8$ modes of fusion. The total statistics to be analyzed can be summarized by $W E R_{\text {pooled }}(\alpha, p, C O M$, data $)$ for

- the performance cost $\alpha \in[0,1]$,

- on the protocol $p=\{\mathrm{Mc}, \mathrm{Ua}, \mathrm{Ud}, \mathrm{P}\}$,

- using any fusion operator $C O M \in\{L R, \operatorname{dep}$, indep, mean $\}$ and

- trained on the data $\in\{$ real,chim $\}$ (real or chimeric).

One can see from Figure 2 that in most cases, the generalization performance on real users was similar whether we used real users for training (thin continuous lines) or chimeric users (thick dashed lines).

We then pooled all measures coming from different fusion operators in order to compare the relative performance between chimericbased fusion models and real user-based fusion models. This relative performance is calculated as

$$
\left(W E R_{\text {chim }}-W E R_{\text {real }}\right) / W E R_{\text {real }} .
$$



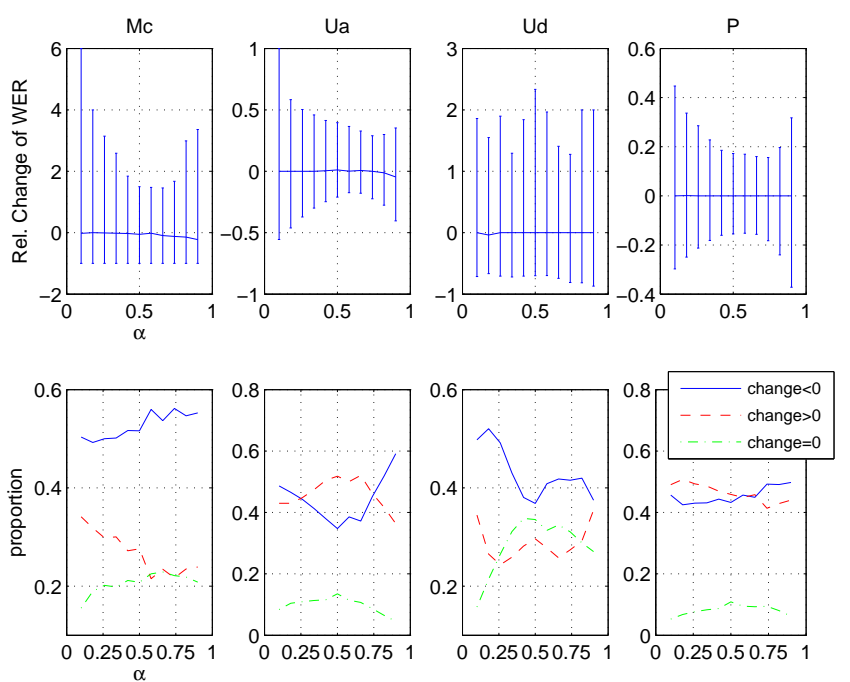

Fig. 3. Upper rows: Relative change of pooled WER on Mc, Ua, $\mathrm{Ud}$ and $\mathrm{P}$ protocols depicted as error bars. Each bar indicates the 2.5th and the 97.5th percentiles and is linked to each other via their respective median. The corresponding lower rows show the proportion of change $<0$ (favors the operators due to chimeric users), $>0$ (favors that due to real users) or $=0$ (i.e., both give exactly the same value).

Hence, a negative change implies that the fusion operator derived from chimeric users improves over its real-user counterpart.

The results, shown in Figure 3, suggest that the generalization performance using chimeric users is not very different from the one using real users (the average relative change is near 0), across different fusion operators. However, for protocol Mc (clean conditions) and Ud (degraded conditions), the fusion operator trained on chimeric users has a higher chance (never less than 50\% and 38\% of the time, respectively) of being consistently better than its real-user counterparts across all cost of $\alpha$ values. Finally, a mixed performance is observed for protocols Ua (adversed) and P (pooled over all three scenarios). In summary, a chimeric database can have a higher chance of improving generalization performance of a fusion operator over not using such information, especially under matched (clean) conditions. It does not make a fusion operator more robust because it is not designed to do so - suggesting that other prior knowledge such as quality information is necessary.

\section{CONCLUSIONS}

Although the use of virtual users is somewhat novel, it should be mentioned that training using virtual samples in machine learning is not new, e.g., [16, 17]. However, different from them, this paper explores how a model can be built using a chimeric database, an approach which to the best of our knowledge, has not been investigated before. One important conclusion from this preliminary study is that a fusion operator derived from a chimeric-user database does not improve nor degrade the generalization performance (on real users) with respect to training it on real users. The advantage, however, is that much more training data can be artificially generated thus in this way it can overcome the lack of training data. This is especially useful when using trainable fusion operators. Note however that, as explained in [1], while chimeric data can be useful to train good fusion operators, the obtained fusion models can only be evaluated on real multimodal biometric data, and not on chimeric data.

\section{REFERENCES}

[1] N. Poh and S. Bengio, "Can Chimeric Persons Be Used in Multimodal Biometric Authentication Experiments?" in LNCS 3869, 2nd Joint AMI/PASCAL/IM2/M4 Workshop on Multimodal Interaction and Related Machine Learning Algorithms MLMI, Edinburgh, 2005, pp. 87100.

[2] A. Ross, A. Jain, and J.-Z. Qian, "Information Fusion in Biometrics," Pattern Recognition Letter, vol. 24, no. 13, pp. 2115-2125, September 2003.

[3] J. Fierrez-Aguilar, J. Ortega-Garcia, J. Gonzalez-Rodriguez, and J. Bigun, "Kernel-Based Multimodal Biometric Verification Using Quality Signals," in Defense and Security Symposium, Workshop on Biometric Technology for Human Identification, Proc. of SPIE, vol. 5404, 2004, pp. 544-554.

[4] J.-L. Dugelay, J.-C. Junqua, K. Rose, and M. Turk, Workshop on Multimodal User Authentication (MMUA 2003). Santa Barbara, CA: no publisher, 11-12 December, 2003.

[5] S. Garcia-Salicetti, M. A. Mellakh, L. Allano, and B. Dorizzi, "A Generic Protocol for Multibiometric Systems Evaluation on Virtual and Real Subjects," in LNCS 3546, 5th Int'l. Conf. Audio- and VideoBased Biometric Person Authentication (AVBPA 2005), New York, 2005, pp. 494-502.

[6] A. Martin, "NIST Year 2001 Speaker Recognition Evaluation Plan," 2001.

[7] C. Marcel, "Multimodal Identity Verification at IDIAP," IDIAP, Martigny, Switzerland, Communication Report 03-04, 2003.

[8] F. Cardinaux, C. Sanderson, and S. Bengio, "User Authentication via Adapted Statistical Models of Face Images," IEEE Trans. on Signal Processing, vol. 54, no. 1, pp. 361-373, January 2006.

[9] S. Pigeon, P. Druyts, and P. Verlinde, "Applying Logistic Regression to the Fusion of the NIST'99 1-Speaker Submissions," Digital Signal Processing, vol. 10, no. 1-3, pp. 237-248, 2000.

[10] C. Bishop, Neural Networks for Pattern Recognition. Oxford University Press, 1999.

[11] T. Hastie, R. Tibshirani, and J. Friedman, The Elements of Statistical Learning. Springer-Verlag, 2001.

[12] A. J. Dobson, An Introduction to Generalized Linear Models. CRC Press, 1990.

[13] M. Figueiredo and A. Jain, "Unsupervised learning on finite mixture models," Pattern Analysis and Machine Intelligence, vol. 24, no. 3, March 2002.

[14] S. Bengio and J. Mariéthoz, "The Expected Performance Curve: a New Assessment Measure for Person Authentication," in The Speaker and Language Recognition Workshop (Odyssey), Toledo, 2004, pp. 279284.

[15] E. Bailly-Baillière, S. Bengio, F. Bimbot, M. Hamouz, J. Kittler, J. Mariéthoz, J. Matas, K. Messer, V. Popovici, F. Porée, B. Ruiz, and J.-P. Thiran, "The BANCA Database and Evaluation Protocol," in LNCS 2688, 4th Int. Conf. Audio- and Video-Based Biometric Person Authentication, AVBPA 2003. Springer-Verlag, 2003.

[16] P. Niyogi, F. Girosi, and T. Poggio, "Incorporating Prior Information in Machine Learning by Creating Virtual Examples," pp. 2196-2209, 1998. [Online]. Available: citeseer.nj.nec.com/niyogi98incorporating.html

[17] N. Poh, S. Marcel, and S. Bengio, "Improving Face Authetication Using Virtual Samples," in IEEE Int'l Conf. Acoustics, Speech, and Signal Processing, Hong Kong, 2003, pp. 233-236 (Vol. 3). 